\title{
Aquaporin 1 is expressed in the human endometrium during normal cycle and increases after mifepristone treatment
}

\author{
ANNA HILDENBRAND ${ }^{1}$, ANNELI STAVREUS-EVERS ${ }^{2}$, P.G.L. LALITKUMAR ${ }^{1}$, \\ SOREN NIELSEN $^{3}$, MIRIAM MINTS $^{4}$ and KRISTINA GEMZELL-DANIELSSON ${ }^{1}$
}

\begin{abstract}
${ }^{1}$ Department of Woman and Child Health, Division of Obstetrics and Gynecology, Karolinska Institutet, Stockholm; ${ }^{2}$ Department of Women's and Children's Health, Uppsala University, Uppsala, Sweden; ${ }^{3}$ The Water and Salt Research Center, Institutes of Anatomy and Experimental Clinical Research, University of Aarhus, Denmark; ${ }^{4}$ Department of Clinical Science, Division of Obstetrics and Gynecology, Karolinska Institutet, Stockholm, Sweden
\end{abstract}

Received January 15, 2008; Accepted February 20, 2008

\begin{abstract}
Aquaporin-1 (AQP1) is involved in the angiogenesis and structural modifications of microvessels and possibly also in the pathogenesis of idiopathic menhorrhagia, where a reduced AQP1 expression is seen in the endometrium. Mifepristone treatment induces reduced menstrual bleeding and amenorrhea and also has a direct effect on endometrial arterioles. Administered with gestagen-only contraceptive methods, antiprogestins improve the bleeding pattern. The objective of this study was to evaluate the AQP1 expression in endometrial blood vessels during normal cycle and after mifepristone treatment. Localization and expression of AQP1 was determined using immunohistochemistry and reverse transcriptase chain reaction (RT-PCR) in 43 biopsies from human endometrium taken during a normal cycle and after mifepristone treatment. AQP1 expression in human endometrial vessels is not cycle dependent and is stronger in capillaries and arteries than in veins. After mifepristone treatment the staining intensity was increased, but not the number of stained vessels. The presence of AQP1 was also confirmed using RT-PCR. The changes in AQP1 expression could contribute to the reduced bleeding seen following mifepristone treatment and could be an effect of either antagonizing progesterone or cortisol.
\end{abstract}

\section{Introduction}

Unscheduled bleeding is a common side effect of gestagenonly contraceptive methods, limiting their acceptability. The bleeding pattern can be improved by combining the treatment with an antiprogestin $(1,2)$. However, the exact underlying

Correspondence to: Anna Hildenbrand, Division of Woman and Child Health, Karolinska Institutet, S-17176 Stockholm, Sweden E-mail: anna.hildenbrand@ki.se

Key words: aquaporin-1, endometrium, water channel, mifepristone, angiogenesis mechanism is not known. Treatment with mifepristone results in an increased expression of estrogen receptors allowing the endometrium to respond to endogenous or exogenous estrogen (3). Mifepristone also directly affects endometrial arterioles in the mid luteal phase (4) as well as inhibits endometrial receptivity and embryo implantation in vivo and in vitro $(5,6)$. When used in low daily doses mifepristone induces amenorrhea in a majority of the women treated (7).

In adult humans physiological angiogenesis, the sprouting of new vessels from existing ones, takes place only in the female reproductive tract and during the wound healing process (8). Endometrial angiogenesis occurs during all phases of the menstrual cycle and in different layers of the endometrium. After the menstrual shedding, new vessels are formed in the remaining superficial basalis layer. During the proliferative phase, angiogenesis takes place at two different locations: throughout the functionalis layer and also directly under the epithelium, where a capillary plexus is formed. These vessels continue growing during the remaining proliferative phase in order to form a network and is sprouted from the spiral arteries at onset of the secretory phase $(9,10)$. During the secretory phase, a development of spiral arteries is observed.

Recently we showed that the expression of aquaporin-1 (AQP1) was reduced in women with idiopathic menhorragia. At present, at least 13 different aquaporins have been identified in mammals (11). AQP1 is an hourglass-shaped membrane integral protein, divided into six membrane-spanning segments (12). This water channel is strongly expressed throughout microvascular endothelia outside of the central nervous system $(13,14)$. Recent experiments in knockout mice revealed a role for AQP1 in angiogenesis, microvascular modifications and endothelial cell migration (15).

The aim of the present study was to examine the effect of mifepristone on the expression of AQP1 in mid-luteal phase endometrial vessels.

\section{Materials and methods}

Endometrial tissue. Endometrial biopsies were obtained from 43 healthy women, with regular menstrual cycles and proven fertility. The mean age was 34 years (range 21-44). None of 
the women had been using steroid hormone contraceptives or an intrauterine device for 3 months or more prior to the study, nor had they been pregnant or had a history of inflammatory pelvic disease within the previous 12 months.

The biopsies originated from the anterior wall of the uterine cavity and were obtained without dilatation using a Randall curette (Stille Werner AB, Stockholm, Sweden). One biopsy was obtained from each woman. From 28 women, the biopsies were taken at a randomly selected time between days $\mathrm{LH}<0$ and $\mathrm{LH}+14$, where the dating refers to the number of days past LH peak (LH peak, day 0). From an additional 15 women, biopsies were obtained in either control cycles (no treatment), or following treatment with a single dose of $200 \mathrm{mg}$ mifepristone administered on day LH+2. The day of LH surge was identified by the participants testing their morning urine using a ClearPlan Home Ovulation Test (Unipath Ltd, Bedford, $\mathrm{UK})$. This test has proven to correlate well with the LH surge determined in peripheral blood (16) $\mathrm{LH}+7$ corresponds to cycle day 21 in a standardized cycle, as per the histology according to Noyes et al (16).

The endometrial samples obtained during the proliferative phase and luteal phase until $\mathrm{LH}+14(\mathrm{n}=28)$, were fixed in $4 \%$ phosphate buffered formaldehyde (Apoteket, Göteborg, Sweden), for a maximum of $24 \mathrm{~h}$ and thereafter stored in $70 \%$ ethanol until being embedded in paraffin. The endometrial samples obtained with or without mifepristone treatment $(n=15)$ were snap frozen in liquid nitrogen. Larger biopsy samples were cut, and one piece was used for RT-PCR analysis. Eight samples were obtained after mifepristone treatment and seven samples were used as control.

The paraffin-embedded biopsies were divided into four groups according to $\mathrm{LH}$ peak $(<\mathrm{LH}+0$ : proliferative phase, $\mathrm{n}=4$; $\mathrm{LH}+0$ to $\mathrm{LH}+5$ : early luteal phase, $\mathrm{n}=8 ; \mathrm{LH}+6$ to $\mathrm{LH}+8$ : mid-luteal phase, $\mathrm{n}=8 ; \mathrm{LH}+9$ to $\mathrm{LH}+14$ : late luteal phase, $\mathrm{n}=8)$.

The Ethics Committee at Karolinska Institutet approved the study and all women gave written informed consent.

Immunohistochemistry. The paraffin-embedded endometrial samples were cut into sections of $4 \mu \mathrm{m}$. The paraffin was removed by rinsing in Bioclear (Bio-Optica, Milan, Italy). The sections were rehydrated using gradient ethanol (99.5, 95 and $70 \%$ ) and finally rinsing in distilled water, and subsequently washing three times in PBS. The frozen samples were mounted in an embedding medium (OTC Compound; Miles Inc, Elkhart, IN, USA) and serially sectioned to $9 \mu \mathrm{m}$ using a Reichert-Jung Cryocut 1800 (Cambridge Instruments $\mathrm{GmbH}$, Nussloch, Germany). The sections were mounted on glass slides, immersed in $2 \%$ paraformaldehyde in PBS, then wrapped in parafilm and stored at $-70^{\circ} \mathrm{C}$ until use. Thereafter all samples were treated in the same way.

Endogenous peroxidase activity was blocked using 3\% hydrogen peroxide in methanol for $30 \mathrm{~min}$ in the dark. Afterwards the sections were washed in PBS/BSA $(0.05 \%)$ and covered with $75 \mu \mathrm{l}$ blocking serum (normal goatserum, 1.5\%, Vector Laboratories Inc., Burlingame, CA, USA) and incubated for $30 \mathrm{~min}$ in a humid chamber. The sections were then incubated with the primary antibody over night at $4^{\circ} \mathrm{C}$ in a humid chamber. The specificity of the AQP1 antibody has been described previously $(17,18)$. After incubation with the primary antibody the slides were washed in PBS/BSA and subsequently incubated with the secondary antibody, a biotinylated goat anti-rabbit antibody (Vector Laboratories), diluted 1:300 with blocking serum, for $30 \mathrm{~min}$ in a humid chamber. Then the slides were washed in PBS/BSA and incubated for $30 \mathrm{~min}$ with horseradish peroxidase-avidinbiotin complex (Vectastain ABC Elite, Vector Laboratories). After washing the slides once more in BSA/PBS the enzyme binding site was visualized by the application of 3,3-diaminobenzidine (a chromogen producing a brown insoluble precipitate when incubated with an enzyme) in $\mathrm{H}_{2} \mathrm{O}_{2}$ (DABkit, Vector Laboratories). The slides were washed in PBS and counterstained with haematoxylin and rinsed in water. Finally the sections were mounted with glycerogelatine (Merck, VWR, Stockholm, Sweden). Negative controls were incubated similarly, but with blocking serum replacing the primary antibody.

$R N A$ isolation and reverse transcriptase chain reaction ( $R T$ $P C R$ ). Total tissue-RNA was isolated using SV Total RNA Isolation System (Promega Corp., Madison, WI, USA) according to the manufacturer's protocol. Total RNA $(2 \mu \mathrm{g})$ was reverse-transcribed using the First-Strand cDNA Synthesis Kit (Pharmacia Biotech AB, Uppsala, Sweden). cDNA (2 $\mu \mathrm{g})$ was then amplified in a total volume of $25 \mu 1$, composed of 10X PCR buffer (100 mM Tris- $\mathrm{HCl}, \mathrm{pH} 8.4,500 \mathrm{mM} \mathrm{KCl})$, $0.5 \mu \mathrm{l} \mathrm{dNTP}, 2 \mu \mathrm{l}$ of each primer $(4 \mu \mathrm{M}), 0.15 \mu \mathrm{l}$ Taq DNA polymerase and $15.85 \mu 1$ sterile water. AQP1 specific primers were generated from known sequences: forward, GTCTTCA TCAGCATCGGTTC and reverse, GTCGGCATCCAGGTC ATACT, as previously described (19). The amplification cycle included denaturation at $95^{\circ} \mathrm{C}$ for $3 \mathrm{~min}, 25$ cycles of amplification at $94^{\circ} \mathrm{C}$ for $30 \mathrm{sec}$, annealing at $64^{\circ} \mathrm{C}$ for $45 \mathrm{sec}$ and extension at $72^{\circ} \mathrm{C}$ for $1 \mathrm{~min}$. Visualization of the RT-PCR products was conducted by electrophoresis using a $1.5 \%$ agarose gel with subsequent treatment with ethidium bromide. As a standard a 100-basepair ladder (Pharmacia Biotech AB) was chosen. In order to exclude the possibility of non-specific amplification, a negative control reaction was included in which no cDNA template was added.

Evaluation of immunohistochemistry and statistics. Evaluation of staining intensity followed using a grading scale of $0-3$, as follows: 0 , no staining; 1 , faint staining; 2 , moderate staining, and 3 , intense staining. The number of stained cells was similar in all biopsies. Two observers, each unaware of the identity of the slides, evaluated the staining intensity. The average value was calculated from the evaluations of the two observers.

Immunostaining of AQP1 was statistically evaluated using the Kruskal-Wallis one-way ANOVA on ranks and all pairwise comparison procedures according to Dunn's method.

\section{Results}

Immunohistochemistry. AQP1 was expressed in the vessels of the human endometrium (Fig.1). There were no statistically significant cycle-dependent changes, either in the intensity of staining, or in the number of vessels stained (Fig. 2A and C). After mifepristone treatment the staining intensity was 

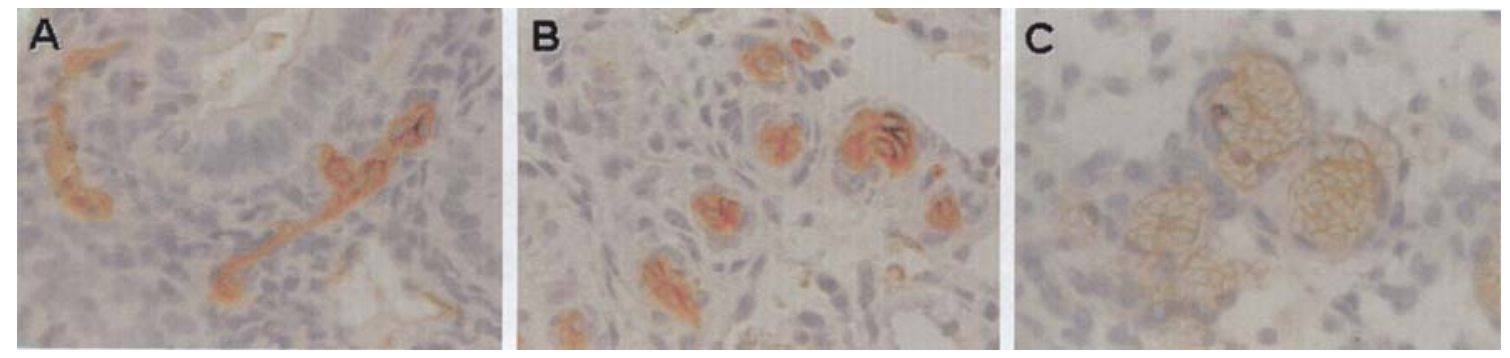

Figure 1. Immunohistochemical staining of AQP1 in endometrium from healthy fertile women. AQP1 is visible in (A) small veins, (B) small arteries and (C) erythrocytes.
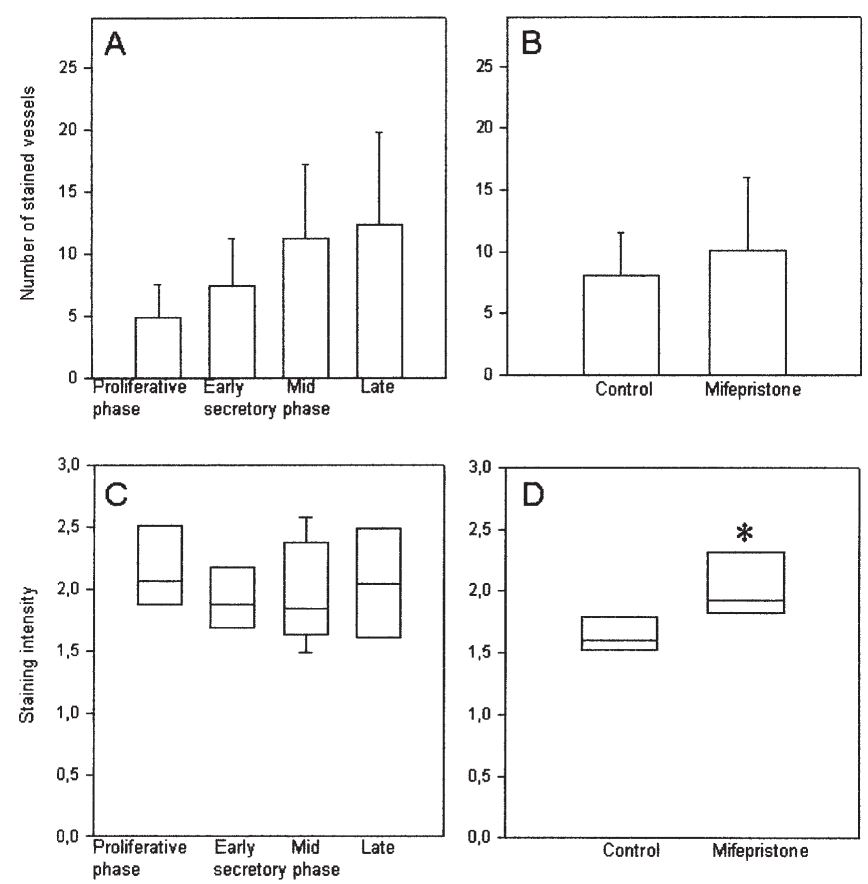

Figure 2. Immunohistochemical data showing staining intensity and number of stained vessels of AQP1 in human endometrium. (A) Staining intensity of AQP1 in endometrium throughout the menstrual cycle. (B) Staining intensity of AQP1 in human endometrium with or without mifepristone treatment on day $\mathrm{LH}+2$. (C) Number of stained vessels in human endometrium throughout the menstrual cycle. (D) Number of stained vessels with or without mifepristone treatment on day $\mathrm{LH}+2$. Statistical differences of $\mathrm{p}<0.05$ are shown.

increased $(\mathrm{p}<0.05)$, but not the number of vessels stained (Fig. 2C and D).

$R T-P C R$. The expression of AQP1 in the human endometrium was verified in RT-PCR, where a positive band of $701 \mathrm{bp}$ was observed (Fig. 3). The optical density ratio was 17.8 , 82.7 and 80.2 for the early, mid- and late secretory phases respectively.

\section{Discussion}

In the human endometrium, angiogenesis is crucial for several events of the normal menstrual cycle. Recent studies in AQP1 knockout mice reveal that AQP1 has a role in angiogenesis and endothelial cell migration (15). We previously demonstrated the aberrant expression of AQP1 in the

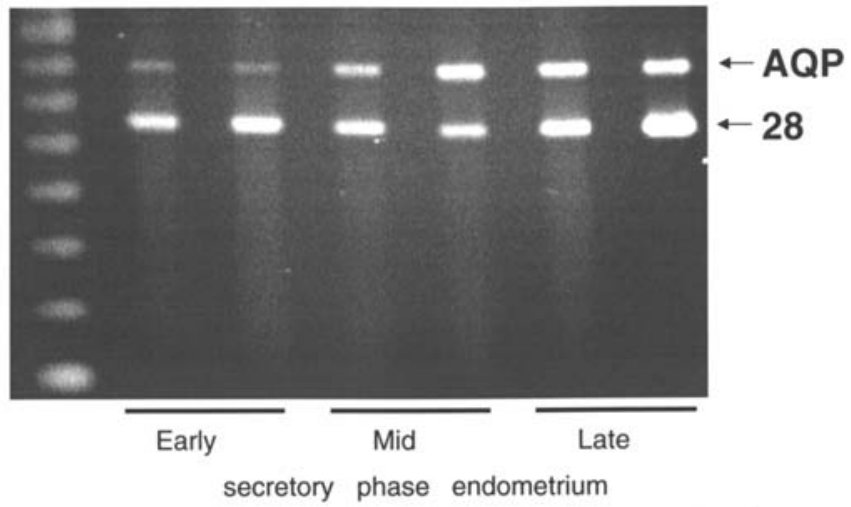

Figure 3. PCR image of AQP1 mRNA in human endometrium.showing early, mid- and late secretory phases.

endometrium of women with menorrhagia compared to healthy women (19). In the present study, we demonstrated that treatment with mifepristone results in the increased expression of AQP1 in endometrial endothelial cells.

Today an increasing number of women request bleedingfree contraceptive methods (20). Unpredictable bleeding is the most common reason for the discontinuation of a gestagenonly contraceptive method. Mifepristone has been shown to have various effects on ovulation and endometrial development depending on the dose given and the duration of the treatment. When given postovulatory once a month it has been shown to act as an effective contraceptive method through the inhibition of endometrial development and receptivity (5). Narverkar et al showed that when given in low daily doses mifepristone induces anovulation as well as affecting endometrial development, resulting in amenorrhea in a high proportion of women (7). The connection between mifepristone-induced amenorrhea, an increased number of glandular glucocorticoid receptors and microvessel density was described in the same study. Therefore it was proposed that the high incidence of amenorrhea after mifepristone treatment might be due to vascular functional regulation (7). Adding mifepristone or other antiprogestins to gestagen-only contraceptive methods significantly improves bleeding pattern (1). A direct effect of mifepristone on endometrial arterioles has been suggested (4). However, while ERß has been shown to be present in endometrial endothelial cells, progesterone receptors (PR) could not be detected in endometrial arterioles. Since AQP1 is involved in endometrial 
angiogenesis and was shown to be reduced in women with menorrhagia, the reduced bleeding could be due to an effect of mifepristone on AQP1 expression.

The presence of AQP1 in the endometrium has been shown in the uterus of both humans and rats $(19,21)$. It has previously been demonstrated that AQP1 is located solely in endothelial cells $(15,22)$. In our previous study, we could not detect any significant differences in vessel staining between cycle day 7 and cycle day 21 . This was confirmed by our present study, where samples obtained during different stages of the secretory phase showed no statistically significant differences, although the mRNA levels of AQP1 increased during the secretory phase. The discrepancy could possibly be explained by technical particularities, e.g. the homogenizing of whole tissue as a preparation for real time PCR, while with the use of immunohistochemistry, variations in specific cell types can be detected.

The female reproductive tract is the only tissue in the adult where physiological angiogenesis takes place $(9,23)$. Angiogenesis, increased vascular permeability and successful remodeling of spiral arteries are important events during endometrial development, implantation and placentation (24). The endometrial capacity for angiogenesis is present throughout the cycle, however, the pattern of angiogenesis is unclear. In artificial cycles in primates, the process of angiogenesis is most intense in the early proliferative phase. In humans, there seems to be a second wave of angiogenesis during the secretory phase as well (25).

The present study shows the distribution of AQP1 in the human endometrium during the normal menstrual cycle and after mifepristone treatment. We have previously reported that AQP1 is present in human endometrium, with an aberrant expression in endometrium from women with menorrhagia, suggesting that AQP1 is important for normal endometrial function (19).

The hormonal regulation of AQP1 is unclear. In rats, both estrogen and progesterone are connected to AQP1 regulation $(21,26)$. In the present study, treatment with mifepristone, a 19-nor steroid that binds with high affinity to the PR, thus inhibiting the effect of endogenous progesterone, increased the staining intensity of AQP1. The increase in AQP1 after mifepristone treatment could imply that progesterone, at least to some extent, is involved in the regulation of AQP1. The highest levels of AQP1 were seen during the late secretory phase, when progesterone levels have declined. However, mifepristone is also responsible for other actions, such as a glycocorticoid effect (27), which might explain the discrepancy between the changes during cycle and the effect of mifepristone. In the present study, the number of stained vessels did not change after a single dose of mifepristone, and it is likely that a longer duration of mifepristone treatment is needed to increase microvessel density (7).

Previous studies showed that other aquaporins, e.g. AQP2 might also be involved in the regulation of water homeostasis in the human endometrium (28). AQP2 is mainly located in the endometrial glandular and luminal epithelium, and could play a role in the regulation of the uterine secretion during the menstrual cycle $(28,29)$. In the mouse uterus, a shift in AQP5 could be observed on the apical surface of the epithelial cells during the time of implantation (21).
A single dose of mifepristone immediately after ovulation has an effect on endometrial development, converting the uterus into a non-receptive stage (30), which is observed when inhibiting the normal downregulation of progesterone receptors in the endometrium (31). Mifepristone is also known to have a high affinity to the glucocorticoid receptor (27). Glucocorticoids suppress angiogenesis in tumor cells (32), and it can be speculated that the effect of mifepristone is due to its action on the glucocorticoid receptor. The precise action of mifepristone in the endometrial epithelial cells and its possible role in the treatment of menorrhagia as well as in reducing menstrual bleeding remains to be further studied.

In conclusion, the presence of AQP1 in human endometrial blood vessels indicates an involvement in the regulation of edema, and also a possible role in the regulation of angiogenesis, as well as endothelial cell migration. Furthermore, the increase in AQP1 staining intensity after mifepristone treatment suggests progesterone as a candidate in AQP1 regulation.

\section{Acknowledgments}

We thank Berit Ståby and Iman El Ali for valuable help with the immunohistochemistry. This study was supported by the Swedish Research Council (2003-3869, 2005-7293), Center for Health Care Sciences Swedish Medical Society, WHO, ALF, the Åke Wibergs Foundation and Karolinska Institutet.

\section{References}

1. Cheng L, Zhu H, Wang A, Ren F, Chen J and Glasier A: Once a month administration of mifepristone improves bleeding patterns in women using subdermal contraceptive implants releasing levonorgestrel. Hum Reprod 15: 1969-1972, 2000.

2. Gemzell-Danielsson K, van Heusden AM, Killick SR, et al: Improving cycle control in progestogen-only contraceptive pill users by intermittent treatment with a new anti-progestogen. Hum Reprod 17: 2588-2593, 2002.

3. Weisberg E, Hickey M, Palmer D, et al: A pilot study to assess the effect of three short-term treatments on frequent and/or prolonged bleeding compared to placebo in women using Implanon. Hum Reprod 21: 295-302, 2006.

4. Johannisson E, Landgren BM, Rohr HP and Diczfalusy E: Endometrial morphology and peripheral hormone levels in women with regular menstrual cycles. Fertil Steril 48: 401-408, 1987.

5. Gemzell-Danielsson K, Swahn ML, Svalander P and Bygdeman M: Early luteal phase treatment with mifepristone (RU 486) for fertility regulation. Hum Reprod 8: 870-873, 1993.

6. Lalitkumar PG, Lalitkumar S, Meng CX, et al: Mifepristone, but not levonorgestrel, inhibits human blastocyst attachment to an in vitro endometrial three-dimensional cell culture model. Hum Reprod 22: 3031-3037, 2007.

7. Narvekar N, Critchley HO, Cheng L and Baird DT: Mifepristoneinduced amenorrhoea is associated with an increase in microvessel density and glucocorticoid receptor and a decrease in stromal vascular endothelial growth factor. Hum Reprod 21: 2312-2318, 2006.

8. Risau W: Mechanisms of angiogenesis. Nature 386: 671-674, 1997.

9. Gargett CE and Rogers PA: Human endometrial angiogenesis. Reproduction 121: 181-186, 2001.

10. Girling JE and Rogers PA: Recent advances in endometrial angiogenesis research. Angiogenesis 8: 89-99, 2005.

11. Agre P and Kozono D: Aquaporin water channels: molecular mechanisms for human diseases. FEBS Lett 555: 72-78, 2003.

12. Agre P, King LS, Yasui M, et al: Aquaporin water channels - from atomic structure to clinical medicine. J Physiol 542: 3-16, 2002.

13. Hasegawa H, Lian SC, Finkbeiner WE and Verkman AS: Extrarenal tissue distribution of CHIP28 water channels by in situ hybridization and antibody staining. Am J Physiol 266: C893-C903, 1994. 
14. Nielsen S, Smith BL, Christensen EI and Agre P: Distribution of the aquaporin CHIP in secretory and resorptive epithelia and capillary endothelia. Proc Natl Acad Sci USA 90: 7275-7279, 1993.

15. Saadoun S, Papadopoulos MC, Hara-Chikuma M and Verkman AS: Impairment of angiogenesis and cell migration by targeted aquaporin-1 gene disruption. Nature 434: 786-792, 2005.

16. Noyes RW, Hertig AT and Rock J: Dating the endometrial biopsy. Am J Obstet Gynecol 122: 262-263, 1975

17. Marchini G, Stabi B, Kankes K, Lonne-Rahm S, Ostergaard M and Nielsen S: AQP1 and AQP3, psoriasin, and nitric oxide synthases 1-3 are inflammatory mediators in erythema toxicum neonatorum. Pediatr Dermatol 20: 377-384, 2003.

18. Smith BL and Agre P: Erythrocyte Mr 28,000 transmembrane protein exists as a multisubunit oligomer similar to channel proteins. J Biol Chem 266: 6407-6415, 1991.

19. Mints M, Hildenbrand A, Lalitkumar LPG, et al: Expression of aquaporin-1 in endometrial blood vessels in menorrhagia. Int $\mathrm{J}$ Mol Med 19: 407-411, 2007.

20. Glasier AF, Smith KB, van der Spuy ZM, et al: Amenorrhea associated with contraception - an international study on acceptability. Contraception 67: 1-8, 2003.

21. Lindsay LA and Murphy CR: Redistribution of aquaporins in uterine epithelial cells at the time of implantation in the rat. Acta Histochem 106: 299-307, 2004.

22. Chen Y, Tachibana O, Oda M, et al: Increased expression of aquaporin 1 in human hemangioblastomas and its correlation with cyst formation. J Neurooncol 80: 219-225, 2006.

23. Folkman J: Angiogenesis in cancer, vascular, rheumatoid and other disease. Nat Med 1: 27-31, 1995.
24. Goodger AM and Rogers PA: Blood vessel growth in the endometrium. Microcirculation 2: 329-343, 1995.

25. Maas JW, Groothuis PG, Dunselman GA, de Goeij AF, Struyker Boudier HA and Evers JL: Endometrial angiogenesis throughout the human menstrual cycle. Hum Reprod 16: 1557-1561, 2001 .

26. Richard C, Gao J, Brown N and Reese J: Aquaporin water channel genes are differentially expressed and regulated by ovarian steroids during the periimplantation period in the mouse. Endocrinology 144: 1533-1541, 2003.

27. Csaba G and Inczefi-Gonda A: Effect of neonatal treatment with mifepristone or tamoxifen on the binding capacity of the thymic glucocorticoid or uterine estrogen receptor of adult rats: data on the mechanism of hormonal imprinting. Life Sci 67: 2531-2537, 2000.

28. Hildenbrand A, Lalitkumar L, Nielsen S, Gemzell-Danielsson K and Stavreus-Evers A: Expression of aquaporin 2 in human endometrium. Fertil Steril 86: 1452-1458, 2006.

29. He RH, Sheng JZ, Luo Q, et al: Aquaporin-2 expression in human endometrium correlates with serum ovarian steroid hormones. Life Sci 79: 423-429, 2006.

30. Csapo AI and Resch B: Prevention of implantation by antiprogesterone. J Steroid Biochem 11: 963-969, 1979.

31. Danielsson KG, Marions L and Bygdeman M: Effects of mifepristone on endometrial receptivity. Steroids 68: 1069-1075, 2003.

31. Yano A, Fujii Y, Iwai A, Kageyama Y and Kihara K: Glucocorticoids suppress tumor angiogenesis and in vivo growth of prostate cancer cells. Clin Cancer Res 12: 3003-3009, 2006. 